\title{
Notch I serves as a prognostic factor and regulates metastasis via regulating EGFR expression in hypopharyngeal squamous cell carcinoma
}

This article was published in the following Dove Press journal: OncoTargets and Therapy

\author{
Jiajun Tian' \\ Xianfang Liu \\ Xiuxiu Liu ${ }^{2}$ \\ Peihang Jing' \\ $\mathrm{NaSa}{ }^{\prime}$ \\ Haibo Wang ${ }^{1,2}$ \\ Wei $X u^{1,2}$
}

'Department of OtorhinolaryngologyHead and Neck Surgery, Shandong Provincial Hospital Affiliated to Shandong University, Jinan 25002I, China; ${ }^{2}$ Shandong Provincial Key Laboratory of Otology, Jinan 250022, Shandong, China

\section{Correspondence: Wei Xu}

Department of OtorhinolaryngologyHead and Neck Surgery, Shandong Provincial Hospital Affiliated to Shandong University, 324 Jingwuweiqi Road, Jinan 25002I, China

Email xuwhns@126.com

Haibo Wang

Shandong Provincial Key Laboratory of Otology, 4 Duanxing West Road, Jinan 250022, Shandong, China Email whbotol I@I63.com
Objective: Hypopharyngeal squamous cell carcinoma (HSCC) remains one of the most lethal malignancies in head and neck. Notch1 has been validated to play prominent roles in the occurrence and development of various types of cancer. The aim of this study was to explore the function and underlying mechanism of Notch1 in HSCC.

Patients and methods: Seventy-one cancer tissue samples and adjacent noncancerous formalin-fixed paraffin embedded tissue specimens were analyzed by immunohistochemistry. As Notch1 is overexpressed in HSCC, we further questioned whether there was a relationship between Notch1 and the clinicopathological characteristics. After confirming the successful knockdown of Notch1 by siRNA, the migration and invasion after gene knockdown were investigated by Transwell chambers. We then tried to identify YBX1 and EGFR expression using real-time PCR (RT-PCR) and Western blot analyses. To further determine whether the downexpression of EGFR was caused by YBX1 and the overexpression of YBX1 was caused by gene amplification, the expression of EGFR was detected by RT-PCR and Western blot assays.

Results: We found that the expression of Notch1 and EGFR in HSCC tissues was upregulated compared with those in the adjacent noncancerous tissues. Further clinicopathological characteristics analysis revealed that the expression of Notch1 was positively correlated with distant metastasis $(P=0.003)$ and tumor differentiation $(P=0.031)$. The high expression of Notch1 is an independent prognostic factor for a poor overall survival in patients with $\operatorname{HSCC}(P=0.015$, $\left.\chi^{2}=10.403\right)$. Knocking down of Notch1 significantly inhibits the migration and invasion of $\mathrm{FaDu}$ cells in vitro. Mechanistic investigation reveals that Notch1 knockdown is found suppressing the expression of EGFR at transcriptional level. Interestingly, we further found that Notch1 knockdown also decreased the expression of YBX1, which is a transcription factor of EGFR. Moreover, the upregulation of YBX1 reverses the suppression of Notch1 on EGFR. Furthermore, forced overexpression of YBX1 induced the invasion of FaDu cells.

Conclusion: Taken together, we found a positively cross-linked role of Notch1 signaling in the outcome of HSCC, providing a novel valuable prognostic marker and potential therapeutic target for the treatment of HSCC patients. Notch1 is a core signaling molecule for regulating migration and invasion via interplaying with EGFR in HSCC cells.

Keywords: hypopharyngeal squamous cell carcinoma, metastasis, Notch1, EGFR

\section{Introduction}

Hypopharyngeal squamous cell carcinoma (HSCC) is one of the most lethal malignancies in head and neck. Currently, the most effective treatment approaches for HSCC are surgical resection, radiotherapy, and chemotherapy, alone or in combination. In spite of the development of various treatments for HSCC, the 5-year survival rate 
of patients with HSCC has not been improved dramatically. Uncontrolled tumor metastasis is one of the main reasons for the poor prognosis of HSCC. Therefore, it is urgent to elucidate new and most effective molecular targeted therapies on metastasis.

The Notch1 signaling pathway is an evolutionarily conserved intracellular signaling, which plays vital roles in both cell differentiation and cell-fate determination processes. ${ }^{1,2}$ Recently, an increasing number of studies have demonstrated that the expression of Notch1 is deregulated in various cancers, such as cervix cancer, ${ }^{3}$ colon cancer, ${ }^{4}$ brain carcinoma, ${ }^{5}$ and head and neck squamous cell carcinomas (HNSCC). ${ }^{6,7}$ Aberrant Notch signaling has been associated with pathogenic conditions such as carcinogenesis, ${ }^{8}$ tumor cell growth, ${ }^{9}$ migration, invasion, ${ }^{9-11}$ and angiogenesis. ${ }^{12}$ Notch expression correlates to the poor prognosis of cancer patients and serves as an early predictive biomarker and a prognostic biomarker for the occurrence and development of HNSCC. ${ }^{8,13,14}$

EGFR is a transmembrane protein receptor with tyrosine kinase activity that triggers numerous signaling pathways. The overexpression of EGFR has been found in many human tumors ${ }^{15}$ and has been reported to be a negative prognostic factor. ${ }^{16,17}$ Activation of the EGFR signal alters protein expression, which results in the enhancement of tumor cell proliferation and suppression of apoptosis, angiogenesis, and invasion. ${ }^{18,19}$ The Notch1 and EGFR pathway are ubiquitous in tumorigenesis and progression, and Notch1 has been shown to upregulate EGFR expression in gliomas. ${ }^{20}$ However, the role of Notch signaling in the metastasis of HSCC remains not fully discovered and the interaction of Notch1 and EGFR expression is not investigated in HSCC.

In this study, we investigated that the expression of Notch1 was upregulated in HSCC cancer tissues and proved significant correlation with clinicopathological features and poor overall survival (OS). Furthermore, we explored a novel cross-talk mechanism between Notch1 and EGFR and investigated the role of this linkage in the metastasis of HSCC.

\section{Patients and methods}

\section{Patients and tissue samples}

Formalin-fixed paraffin-embedded tissue specimens were obtained from 71 patients who suffered from HSCC and underwent surgery at the Department of Otorhinolaryngology-Head and Neck Surgery in Shandong Provincial Hospital Affiliated to Shandong University, Jinan, China. The inclusion criteria of this study were as follows: 1) previously untreated hypopharyngeal cancer, 2) histologically proven squamous cell carcinoma, and 3) curative-intent surgical treatment and postoperative adjuvant radiotherapy. The exclusion criteria were as follows: 1) recurrent tumor, 2) distant metastasis at the initial visit, and 3) treatment for other cancers. Of the 71 patients, 67 patients were men and four patients were women. The median age of patients was 57.86 years (range, 44-72 years). Seventy-one HSCC cancer tissue samples were collected from June 2010 to September 2014. Adjacent noncancerous tissue samples were collected at the same time. None of the patients had received chemical therapy or radiotherapy prior to surgery. All patients were followed up by telephone or history inquiry; clinicopathological, demographic, and radiological data were collected from the electronic records. Distant metastases were defined as follows: all cases are recommended to undergo enhanced computed tomography (CT) or brain contrast-enhanced magnetic resonance imaging. According to the diagnostic criteria of lung metastases or brain metastases, 30 cases with lung/brain metastases were included and 41 cases with nonlung/brain metastases were matched with metastases cases. The study was approved by the ethics committee of Shandong University (Jinan, China). All patients received detailed pretest counseling and provided written informed consent for the test.

\section{Immunohistochemical (IHC) analysis}

The quality of tumor/adjacent noncancerous tissue was monitored under the guidance of a clinical pathologist. All tissue slides were deparaffinized and rehydrated, and antigen retrieval was done by incubating the slides at $95^{\circ} \mathrm{C}$ for 20 minutes in Target Retrieval Solution (ZSJB-BIO) according to the manufacturer's instructions. Slides were washed three times with PBS and then incubated with anti-Notch1 polyclonal antibody (diluted 1:200; OriGene Technologies, Inc., Rockville, MD, USA) or mouse antiEGFR monoclonal antibody (diluted 1:1,000; Santa Cruz Biotechnology, Inc., Dallas, TX, USA) at $4{ }^{\circ} \mathrm{C}$ overnight and washed twice with $\mathrm{PBS}$ prior to incubation with a secondary antibody at room temperature for 30 minutes. The slides were examined using a light microscope. Histopathological diagnosis of all tissue samples was performed by two clinical pathologists.

\section{Cell culture and siRNA transfection}

The human hypopharyngeal carcinoma FaDu cell line was obtained from the American Type Culture Collection (ATCC, Manassas, VA, USA). The FaDu cells were grown at $37^{\circ} \mathrm{C}$ at $5 \% \mathrm{CO}_{2}$ in DMEM (Thermo Fisher Scientific, Waltham, 
MA, USA) supplemented with $10 \%$ fetal calf serum (Thermo Fisher Scientific), $100 \mathrm{U} / \mathrm{mL}$ of penicillin, and $0.1 \mathrm{mg} / \mathrm{mL}$ of streptomycin. FaDu cells seeded as single cells $\left(3 \times 10^{5} /\right.$ dish $)$ were left for 1 day in proliferation medium before treating them with the siRNA, either singly or in combination, for 1-2 days.

The experiments on RNA interference were performed using Notch1 siRNA (si-Notch1). si-Notch1 and siRNA negative control (siRNA-NC) were purchased from GenePharma (Shanghai, China). FaDu cells were transiently transfected with $100 \mathrm{nM}$ siRNA. The sequences for si-Notch1 were as follows: sense strand $5^{\prime}$-UCG CAU UGA CCA UUC AAA CUG GUGG-3' and antisense strand 5'-CCA CCA GUU UGA AUG GUC AAU GCGA-3'. FaDu cells were transfected with siRNA using the Lipofectamine 3000 Reagent (Thermo Fisher Scientific) according to the manufacturer's protocol. Cells were harvested 1-2 days after transfection.

\section{RNA extraction and quantitative real- time PCR (RT-PCR)}

Total RNA was extracted using the TRIzol reagent (Thermo Fisher Scientific). cDNAs were obtained using the RevertAid First Stand cDNA Synthesis kit (Thermo Fisher Scientific). RT-PCR was performed in triplicate using the SYBR Premix Ex Taq (Takara, Dalian, China), and $\beta$-actin was used as an internal control. The PCR primers used for quantitative RT-PCR were as follows: Notch1, 5'-GAA TGG TCA ATG CGA GTG G-3' (forward) and 5'-TGG CAG CAA GGC TAC TGT G-3' (reverse); EGFR, 5'-TTG CCG CAA AGT GTG TAA CG-3' (forward) and 5'-GTC ACC CCT AAA TGC CAC CG-3' (reverse); YBX1, 5'-AAG TGA TGG AGG GTG CTG AC-3' (forward) and 5'-TTC TTC ATT GCC GTC CTC TC-3' (reverse); and $\beta$-actin, 5'-CCA ACC GCG AGA AGA TGA-3' (forward) and 5'-CCA GAG GCG TAC AGG GAT AG-3' (reverse).

\section{Western blots}

Afterward, cells were collected and washed with PBS with protease inhibitors before protein extraction. Total cell lysates $(20 \mu \mathrm{g})$ were assessed by Western blot using standard procedures. Protein levels in all experiments were normalized to $\beta$-actin. The following primary antibodies were used: Notch1 (diluted 1:1,000; Cell Signaling Technology, Danvers, MA, USA), EGFR (diluted 1:10,000; Santa Cruz Biotechnology, Inc.), YBX1 (diluted 1:1,000; Cell Signaling Technology), p-YBX1 (diluted 1:1,000; Cell Signaling Technology), and $\beta$-actin (diluted 1:40,000; Sigma-Aldrich Co., St Louis, MO, USA).

\section{Cell migration and invasion assays}

To examine the function of Notch1 in HSCC motility, we used transwell chambers. For transwell migration assays, FaDu cells in exponential growth were harvested, washed, and suspended in DMEM without FBS. Cells $\left(1 \times 10^{5} / 100 \mu \mathrm{L}\right)$ were seeded into the upper chambers ( $8 \mu \mathrm{m}$ pore size) in 24-transwell cell culture dishes. The lower chamber was filled with $600 \mu \mathrm{L}$ of DMEM with $10 \%$ FBS. Cells were permitted to migrate for 24 hours. After the incubation, stationary cells were removed from the upper surface of the membranes. The cells that had migrated to the lower surface were fixed and stained with $0.1 \%$ crystal violet, counted from five random fields, and averaged. Experiments were repeated three times.

Cell invasion was investigated using the same protocol as for cell transwell migration but with the use of Matrigel (BD Biosciences, San Jose, CA, USA) precoated cell culture inserts. In brief, $1 \times 10^{5}$ cells in $100 \mu \mathrm{L}$ of DMEM without FBS were seeded into the upper chambers of $8 \mu \mathrm{m}$ pore transwells. For the assay of invasion through a Matrigel barrier, cells were permitted to invade for 36 hours. Invasive cells were fixed, stained, counted, and averaged.

\section{Statistical analysis}

All data in the present study were obtained from three independent experiments and are expressed as mean $\pm \mathrm{SD}$. Mann-Whitney $U$-test was performed to compare Notch1 and EGFR expression in the tumor tissues and paired adjacent normal tissues. Cell proliferation, migration, and invasion assays were analyzed by Student's $t$-test. One-way ANOVA, Wilcoxon rank-sum test, rank correlation, Chi-squared test, and survival analysis (Kaplan-Meier) were used to evaluate the differences between multiple comparisons. All analyses were performed using SPSS 17.0 software (SPSS Inc., Chicago, IL, USA). $P<0.05$ was considered a statistically significant difference.

\section{Results IHC expression of Notch I and EGFR in HSCC}

Aberrant Notch1 is highly associated with oncogenesis in many types of human cancers. To investigate whether the level of Notch1 expression affects tumorigenesis of HSCC, the expression of Notch1 in HSCC was determined by IHC analysis (Figure 1). According to the results of staining evaluation, the expression of Notch1 can be seen from both cytoplasm and nuclei of tumor cells in HSCC. Notch1 was found in the majority of HSCC cancer tissues evaluated, and 
A

Protein expression of Notch1 in patients with HSCC
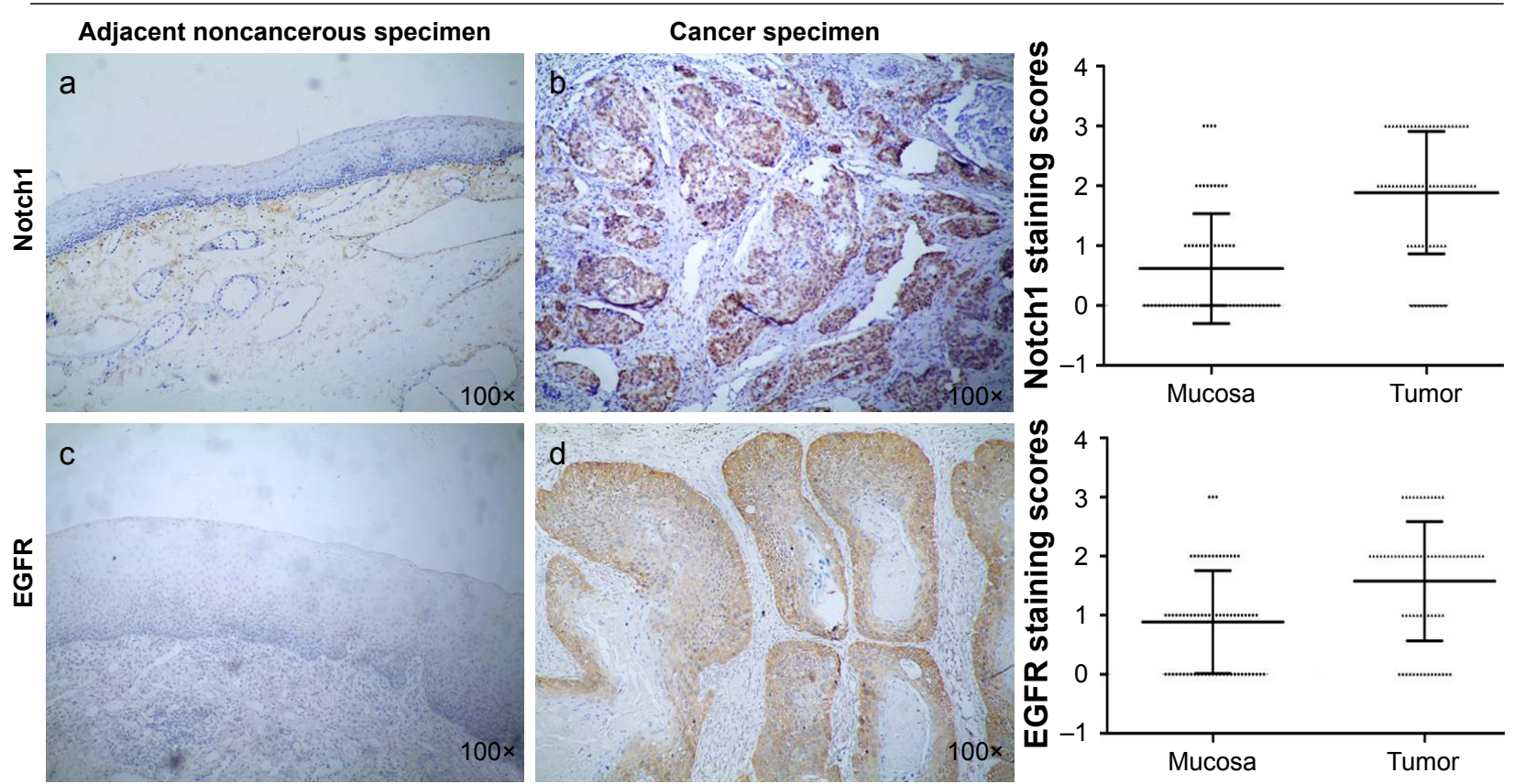

B

Notch1 expression in various HSCC tumor specimens
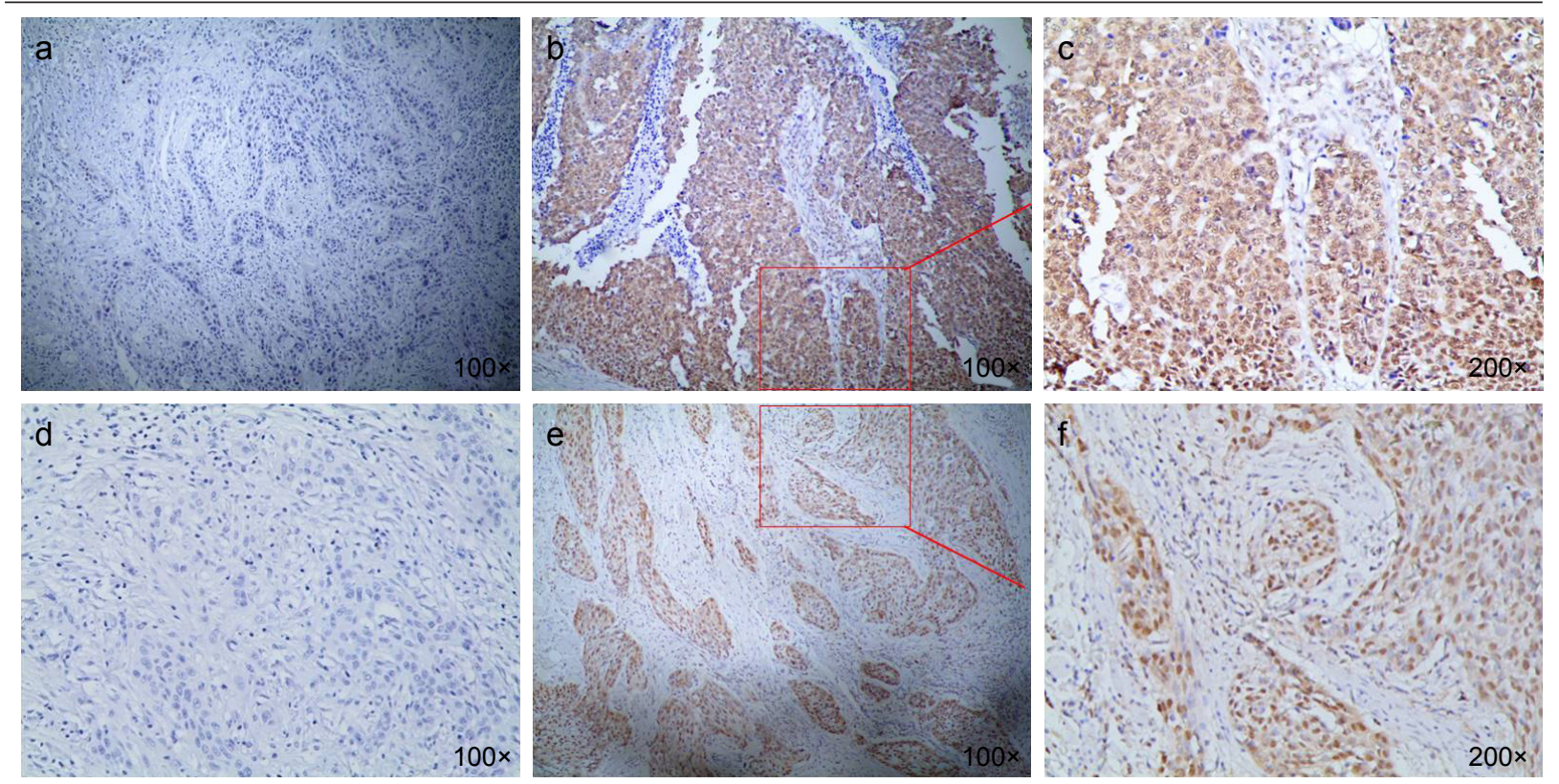

Figure I Immunohistochemical images for the expression of NotchI in HSCC tumor and adjacent noncancerous specimens.

Notes: (A) Representative images of Notchl expression in tumor and adjacent noncancerous specimens. (a) Notch I expression in adjacent noncancerous specimens $(100 \times)$. (b) Brown staining indicates Notch I positivity expression in cancer tissue (I00x). (c) EGFR expression in adjacent noncancerous specimens (I00X). (d) Brown staining indicates EGFR positivity expression in cancer tissue $(100 \times)$. (B) Representative images for low expression ( $-/+$, two cases on the left; a and d), and high expression $(++/++, 2$ cases on the middle; $b$ and e) of Notchl in various tumor samples. A segment of the high expression is magnified at $200 \times(c$ and $f)$.

Abbreviation: HSCC, hypopharyngeal squamous cell carcinoma.

the expression of Notch1 was markedly higher in cancer tissues than in adjacent hypopharyngeal epithelium tissues $(P<0.001)$ (Figure 1A). The number of Notch1 high expression in tumor tissue was $47 / 71$ cases $(66.2 \%)$ (Figure 1B) compared with $14 / 71$ cases $(19.8 \%)$ in adjacent normal tissues.

The EGFR protein expression of all HSCC tissues were assayed by IHC method. The immunostaining of EGFR was 
found in the cytoplasm and membrane. We demonstrated that HSCC tumor tissues exhibited significantly higher EGFR expression compared with normal tissues $(P<0.001$; Figure $1 \mathrm{~A}-\mathrm{c} / \mathrm{d})$.

\section{High expression of Notch I is significantly associated with distant metastasis in patients with HSCC}

As shown in Table 1, the association between Notch1 expression and clinicopathological characteristics, including tumor location, differentiation, tumor $\mathrm{T} / \mathrm{N}$ stage, and distant metastasis, was evaluated. The results showed that high level of Notch1 expression was positively correlated with the status of histological differentiation of HSCC $(P=0.031)$. Present data show that the differences between Notch1 expression and the status of clinical stage as well as $\mathrm{N}$ classification were not significant $(P>0.05)$. Additionally, there was no correlation between Notch1 overexpression and tumor location $(P>0.05)$.

Pulmonary and brain metastases are the most frequent distant metastases of HSCC patients. In the HSCC patients, Notch1 expression significantly correlated with distant metastasis $(P=0.003)$. Of the 48 samples $++/+++$ positive for Notch1 in cancer tissue of HSCC patients, 24 (50.00\%) samples were also found to be positive for distant metastases. Correspondingly, of the 23 samples -/+ positive for Notch 1 expression, six $(26.09 \%)$ samples were also found to be positive for distant metastases.
The overexpression of Notch I is related to unfavorable prognosis for HSCC

The overall 3- and 5-year survival rates were 54.93 and $35.43 \%$, respectively, in these patients. To further evaluate the prognostic value of Notch1 in HSCC patients, the Kaplan-Meier estimator model analysis was performed (Figure 2). The overexpression of Notch1 is associated with a much poorer prognosis $\left(P=0.015, \chi^{2}=10.403\right)$. Patients with higher Notch1 expression had a significantly shorter OS time than patients with low Notch1 expression. The 3-year OS rates in patients with low Notch1 expression and high expression were 78.26 and $43.75 \%$, respectively. The high expression of Notch1 was found within as much as $84.38 \%$ $(27 / 32)$ of patients with worse survival status.

\section{Notch I knockdown inhibited the migration and invasion of in vitro cultured FaDu cells}

To verify the efficiency of knockdown, we examined the relative protein and mRNA levels of Notch1 expression in siRNA-NC and si-Notch1 FaDu cells by Western blot and RT-PCR analyses. As shown in Figure 3A, FaDu-control cells expressed more Notch1 compared with parental $\mathrm{FaDu}$ siRNA cells, suggesting effective suppression of Notch1 gene expression by si-Notch1 $(P<0.05)$.

Cell counting kit-8 (CCK-8) assay was used to investigate the influence of Notch1 expressed on the growth of FaDu cells. After treating FaDu cells with si-Notch1 and

Table I The correlations between Notch I expression and HSCC patients' clinicopathological characteristics of the study group

\begin{tabular}{|c|c|c|c|c|}
\hline \multirow[t]{2}{*}{ Features } & \multirow{2}{*}{$\begin{array}{l}\text { Number } \\
\text { of cases }\end{array}$} & \multicolumn{2}{|c|}{ Notch I expression } & \multirow[t]{2}{*}{$P$-value } \\
\hline & & Low $(n=23)$ & High $(n=48)$ & \\
\hline Age (years) & $57.86(44-72)$ & & & \\
\hline Gender (male/female) & $67 / 4$ & & & \\
\hline Location & & & & 0.139 \\
\hline Pyriform sinus & 56 & 18 & 38 & \\
\hline Posterior pharyngeal wall & 11 & 5 & 6 & \\
\hline Postcricoid & 4 & 0 & 4 & \\
\hline Histological grade & & & & 0.031 \\
\hline Well differentiation & 3 & 3 & 0 & \\
\hline Intermediately poor differentiation & 68 & 20 & 48 & \\
\hline T staging & & & & 0.090 \\
\hline TI-2 & 11 & I & 10 & \\
\hline T3-4 & 60 & 22 & 38 & \\
\hline $\mathrm{N}$ staging & & & & $>0.05$ \\
\hline No & 10 & 3 & 7 & \\
\hline $\mathrm{N}+$ & 61 & 20 & 41 & \\
\hline Metastasis & & & & 0.003 \\
\hline No & 41 & 6 & 24 & \\
\hline Yes & 30 & 17 & 24 & \\
\hline
\end{tabular}

Abbreviation: $\mathrm{HSCC}$, hypopharyngeal squamous cell carcinoma. 


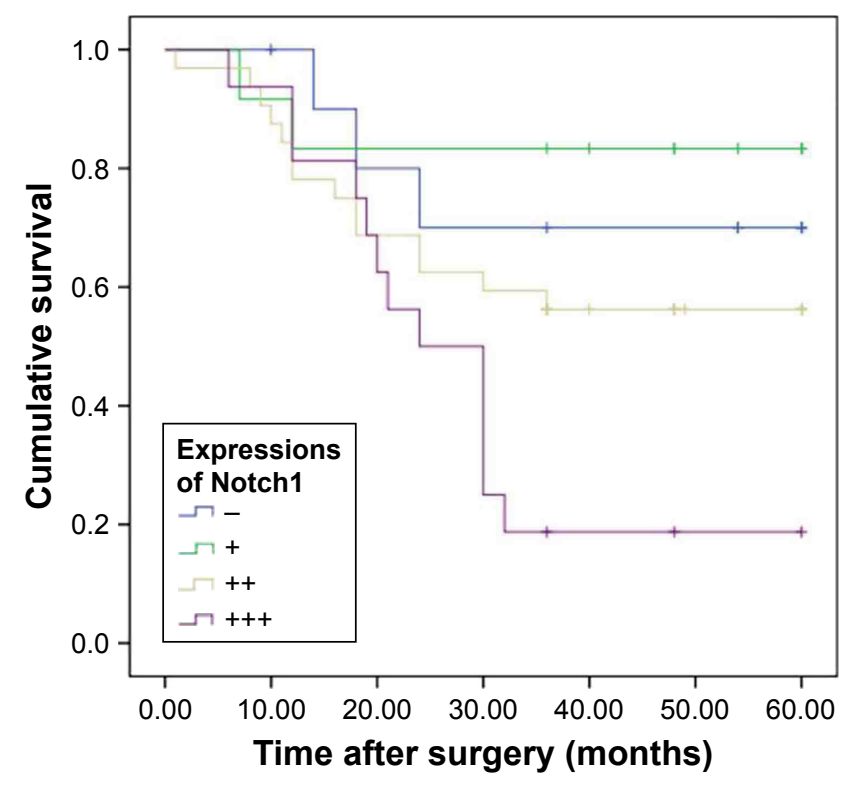

Figure 2 The Notch I expression was an independent prognostic factor for overall survival by multivariate analyses.

Note: Furthermore, compared with low expression cases $(-/+)$, the overexpression $(++/+++)$ of Notchl in HSCC tissues had a poor prognosis with a low overall survival rate.

Abbreviation: HSCC, hypopharyngeal squamous cell carcinoma.

siRNA-NC for 24 and 48 hours, si-Notch1 treatment for these two time points did not reduce the proliferation of $\mathrm{FaDu}$ cells compared with control group ( $P=0.93$ vs 0.22 , respectively) (Figure 3B). Because of these results, cell invasion assay and migration assay were performed with si-Notch1 or siRNA-NC to ignore the influence of cell viability.

To address whether Notch1 participates in the invasion and migration of HSCC, we first examined the migration capability of $\mathrm{FaDu}$ cells after verifying the downregulation of Notch1 expression by siRNA. By the migration assay, the mean number of migratory Notch1-knockdown cells was compared with that of the controls. We found that si-Notch1-transfected $\mathrm{FaDu}$ cells exhibited a significant decrease in the migratory potential as compared to the control cells (Figure $3 \mathrm{C}-\mathrm{a} / \mathrm{b}$, $P<0.01)$. The invasive characteristics of Notch1-knockdown cells were then investigated using invasion assay. Following cell invasion assay, we found that silencing Notch1 also inhibited the invasion capability in those si-Notch1transfected cells (Figure $3 \mathrm{C}$-c/d, $P<0.01$ ). These results indicated that downregulated Notch1 may have a negative correlation with HSCC cell migratory and invasive potential.

\section{Downregulation of Notch I suppressed} the activation of intracellular YBXI-EGFR signaling pathways

To begin uncovering mechanisms mediating the influence of Notch1 signaling on the metastasis of HSCC, we initially investigated the expression of EGFR. Following the cells transfecting with si-Notch1, we detected the expression of EGFR in the mRNA and protein levels in different treatments of cells. As shown in Figure 4A, the level of EGFR mRNA expression was decreased significantly following siRNA transfection. This result was further confirmed by Western blot analysis (Figure $4 \mathrm{C}, P<0.05$ ).

It has been reported that YBX1 can directly bind to the regulatory regions of EGFR. To further examine whether the YBX1 was involved in the Notch1-induced EGFR regulation in FaDu cells, we employed RT-PCR and Western blot analyses to investigate the expression of YBX1. We identified that the expression of YBX1 was markedly inhibited both at the mRNA and protein levels in si-Notch1 FaDu cells compared with control group (Figure 4B and C, $P<0.05$ ). In addition, the results of our study showed that Notch1 knockdown decreased the expression of p-YBX1 $(P<0.05)$. However, the difference between the loss in pYXB1 with respect to total YXB1 was not significant.

\section{YBXI overexpression reverses the decrease in EGFR after Notch I knockdown and upregulates the invasion of FaDu cells}

YBX1 is well understood to regulate EGFR expression. To further investigate the possible role of YBX1 in Notch1associated signaling pathways, we next addressed whether improving YBX1 activation with the gene overexpression would impact the expression of its target gene EGFR,. As shown in Figure 5, the relative protein expression levels of YBX1 in FaDu cells co-transfected with si-Notch1 and YBX1 expression vectors were significantly higher than levels in control cells $(P<0.05)$. To further support this observation, we examined $\mathrm{p}-\mathrm{YBX} 1$ protein level. The expression of p-YBX1 protein level was elevated after 48 hours treatment with YBX1 overexpressing system compared with the control groups. Similarly, 48 hours treatment with the YBX1 overexpressing system resulted in a marked increase in EGFR protein levels.

We further examined the invasion capability of $\mathrm{FaDu}$ following cotransfection. Compared with negative control groups, after cotransfection with si-Notch1 and YBX1 expression vectors for 36 hours, the inhibition of cell invasion in the co-transfection group was reversed $(P<0.01)$. Invasion analysis demonstrated that the invasive rate increased to a higher extent in the co-transfection group relative to the other group at 48 hours after co-transfection. Present data further confirm that the downregulation of Notch1 was able 

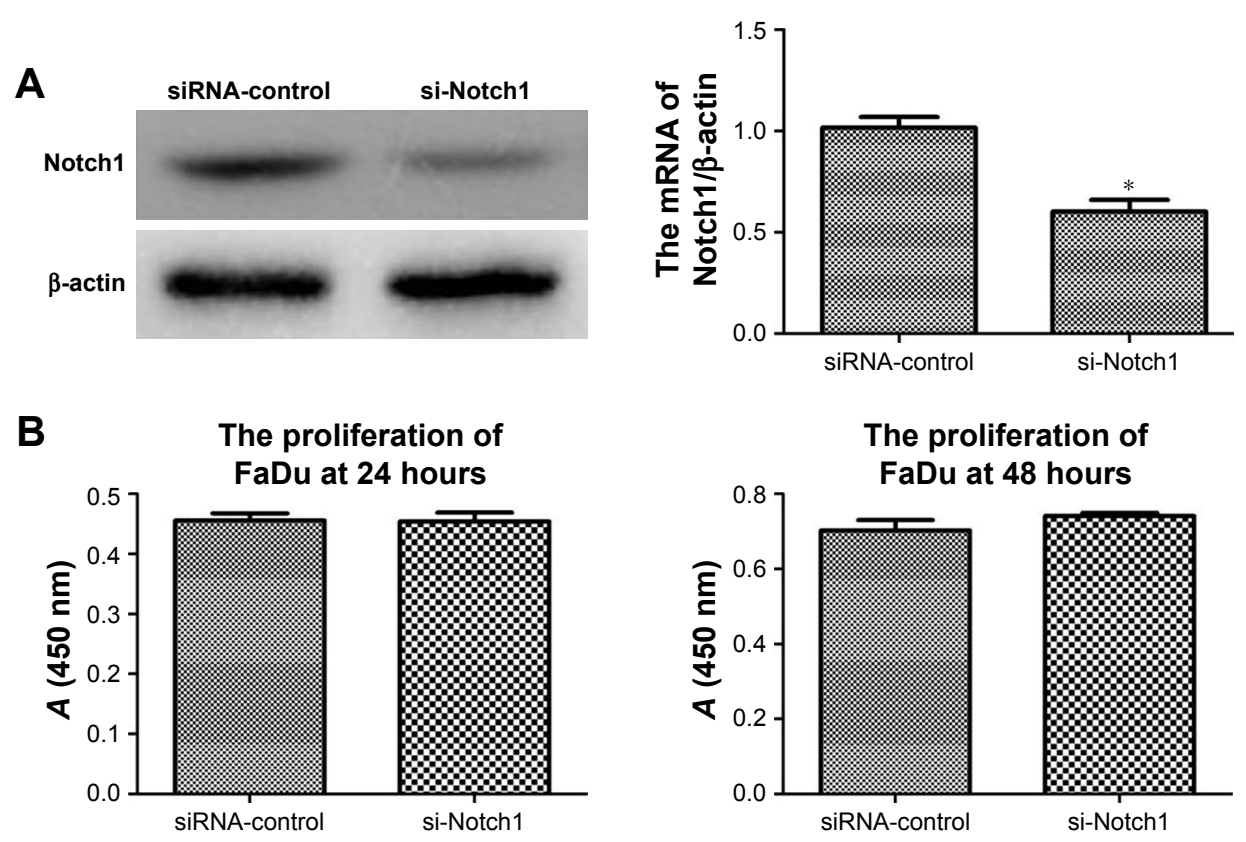

C SiRNA-control
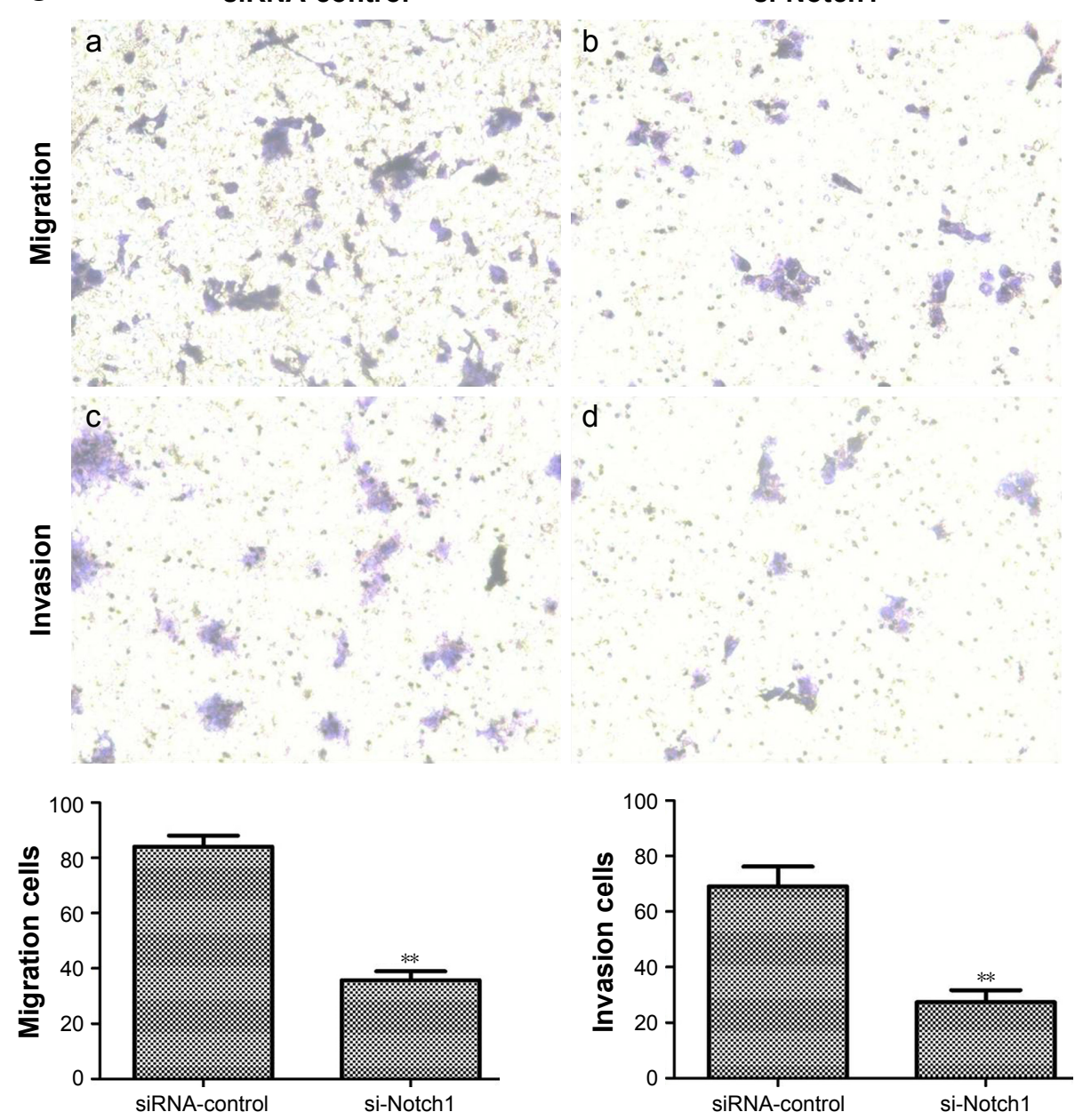

Figure 3 The downregulation of Notchl inhibited the metastasis of FaDu.

Notes: (A) The efficiency of knock down were confirmed by western blot and real time PCR. In comparison with cells transfected with negative control siRNA, transfection with siRNA targeted against the Notch I significantly inhibited Notch I expression in FaDu cells $(* P<0.05)$. (B) The proliferation of si-Notch I or siRNA-NC-treated FaDu cells for 24 and 48 hours evaluated by CCK-8 assay $(P>0.05)$. (C) Transwell assay was employed to examine the invasive and migrating ability of transfected cells (I00 $\times$ ). (a) The migration of control group. (b) The migration of si-Notch I group. (c) The invasion of control group. (d) The invasion of si-Notch I group ( $* * P<0.0 \mathrm{I}$ ).

Abbreviations: CCK-8, cell counting kit-8; si-Notch I, NotchI siRNA; siRNA-NC, siRNA negative control. 
A

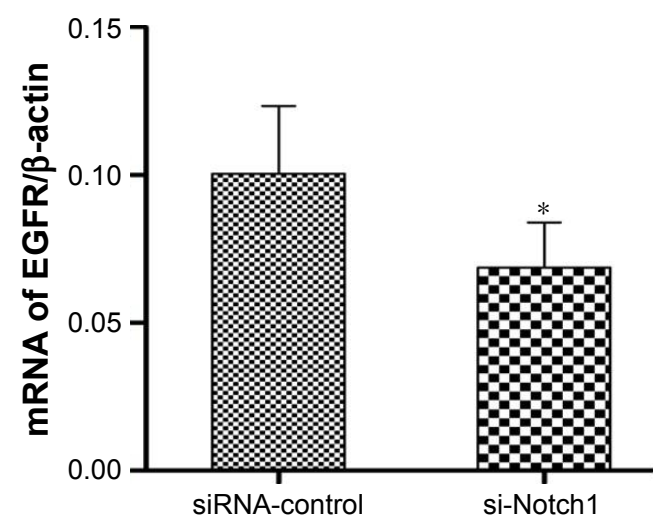

C

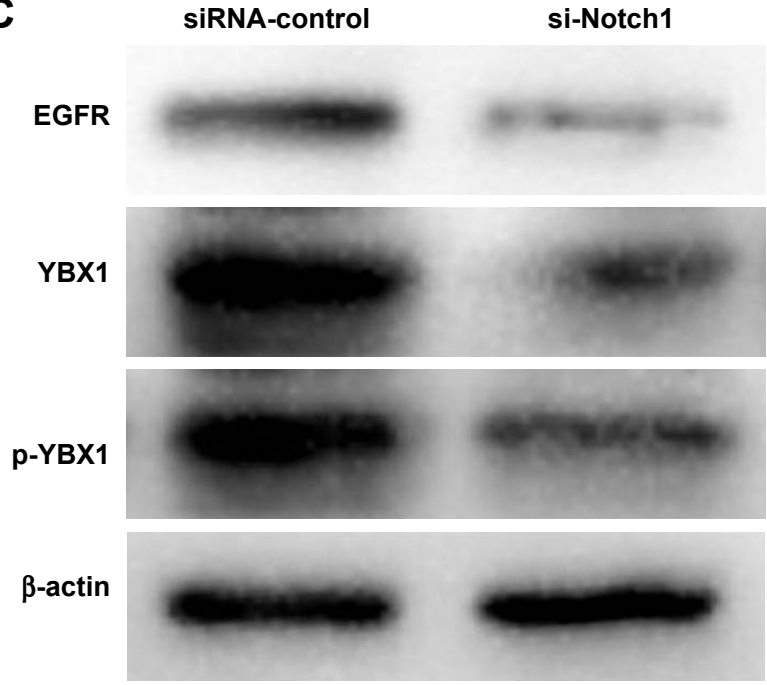

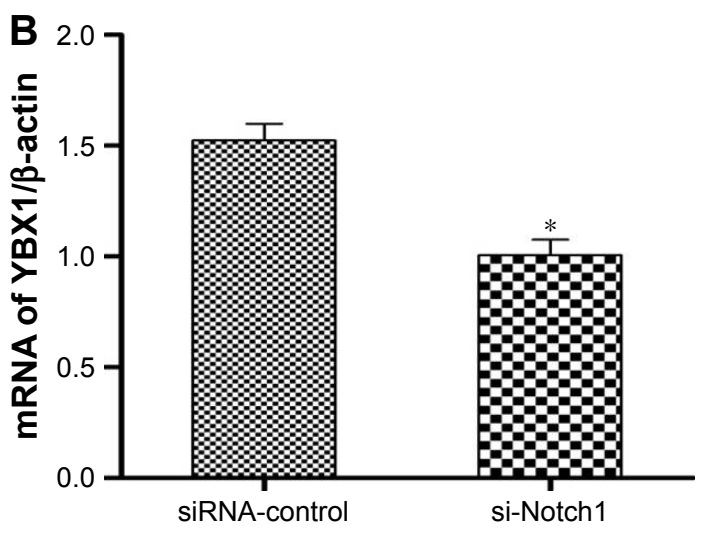

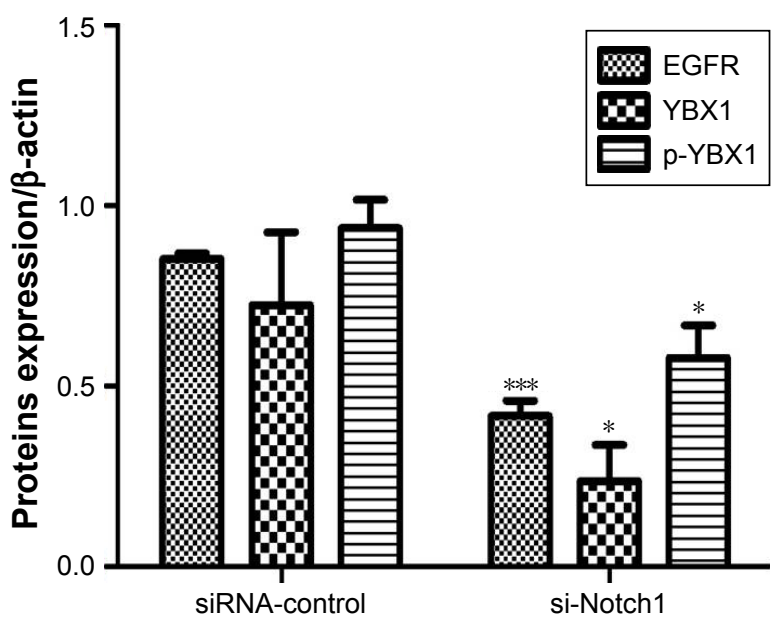

Figure 4 The expression of EGFR and YBXI were significantly inhibited after si-Notch I transfection.

Notes: $(\mathbf{A}$ and $\mathbf{B})$ The RNA levels of EGFR and YBXI by real-time PCR assay $(* P<0.05)$. (C) The protein levels of EGFR, YBXI, and $P-Y B X I$ by $W$ estern blot assay $(* P<0.05$, $* * * P<0.001)$.

Abbreviation: si-Notchl, Notchl siRNA.

to inactivate EGFR by regulating YBX1, which resulted in the inhibition of HSCC cell invasion and migration.

\section{Discussion}

Although surgical and treatment measures have developed overtime, the long-term survival rate of HSCC patients has not been improved dramatically. Based on clinical observations, the lack of effective treatment for the metastasis of HSCC has become a major obstacle in improving survival rates. Therefore, it is urgent to elucidate the molecular mechanisms of postoperative metastasis and explore new therapeutic strategies. Over the past decade, the tumorigenic potential of aberrant Notch1 signaling has been implicated in a wide variety of epithelial cancer types. The Notch signaling pathway is involved in the carcinogenesis, progress, invasion, and neovascular formation of many malignant tumors. ${ }^{20-22}$ However, the role of Notch signaling in HNSCC remains inadequately defined and is often controversial. Some studies implicated Notch as a pro-oncogenic signaling pathway in HNSCC. ${ }^{23,24}$ In contrast, other studies suggested that Notch is tumor suppressive, Agrawal et $\mathrm{al}^{25}$ reported that Notch1 may function as a tumor suppressor gene rather than an oncogene in HNSCC.

It has been well identified that Notch1 is related to a wide diversity of essential biological processes in tumor, such as histological differentiation, tumor invasion, and lymph node status. The relationship between Notch1 expression levels and certain clinicopathological parameters was evaluated. We found a significant overexpression of Notch1 in tumor samples of HSCC compared with normal hypopharyngeal epithelium samples. Additionally, further clinical feature analysis found that the overexpression of Notch1 was significantly associated with poor differentiation of tumor tissue. Previous report showed that Notch1 expression level in tumor samples is directly correlated with the invasiveness of the malignant lesions, represented by tumor stage/grade. ${ }^{20,23}$ 

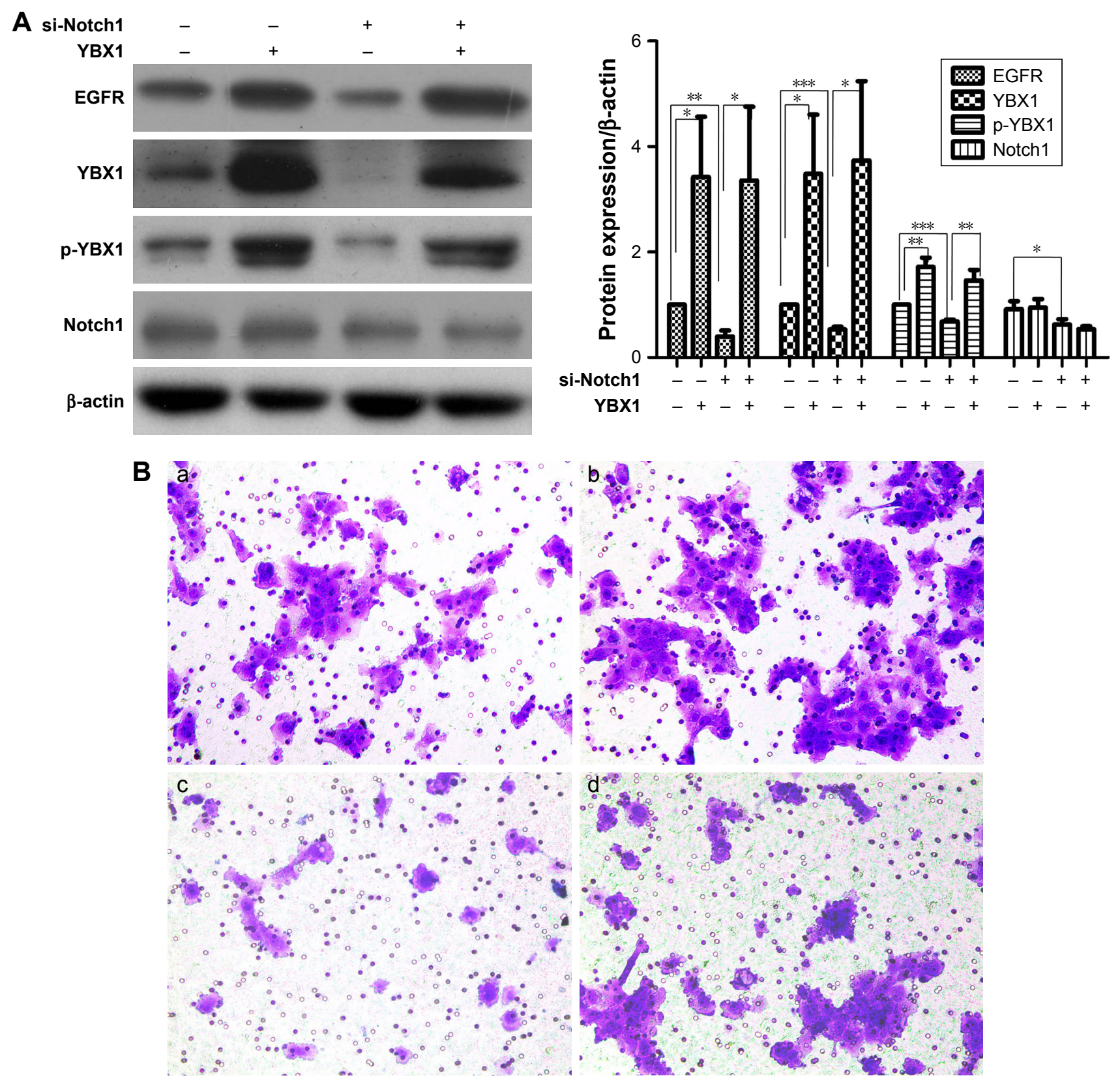

Figure 5 Ectopic expression of YBXI resulted in substantial reversion on the effectiveness of Notch I knockdown.

Notes: (A) Further evidence showed that YBXI upregulation antagonized Notchl knockdown-induced inhibition on EGFR. The Western blot assay was used to record EGFR, YBXI, and $\mathrm{P}$-YBXI expression in FaDu cells co-transfected with si-NotchI and YBXI genes $(* P<0.05$, $* * P<0.01$, and $* * * P<0.00 I)$. (B) Representative images of invaded FaDu cells when co-cultured with or without si-Notch I and siRNA-NC in invasion assay (magnification, 20X). Forced YBXI expression promoted the invasion of FaDu (**P<0.0I). (a) The cells were treated with siRNA-NC and PCDNA3.I. (b) The cells were treated with siRNA-NC and PCDNA3.I-YBXI. (c) The cells were treated with si-Notch I and PCDNA3.I. (d) The cells were treated with si-Notch I and PCDNA3.I-YBXI.

Abbreviations: si-Notch I, Notch I siRNA; siRNA-NC, siRNA negative control.

Interestingly, the differences of Notch1 expression correlated to tumor invasion and lymph node status were not significant in the our present data.

Prognostic biomarker provides useful information for clinical treatment and it is conducive for identifying patients who have a higher risk of recurrence or poor outcome. In the current study, the potential role for Notch1 as a HSCC prognostic biomarker was demonstrated. Distant metastasis is one important aspect of tumor recurrence, accounting for the main cause for the death of HSCC patients. Our results showed that the elevated expression of Notch1 was much more in the distant metastasis group than in the nondistant metastasis group. These data indicated that Notch1 may participate in the development of postoperative distant 
metastasis of HSCC. Further clinical feature analysis found that the analysis of Notch1 expression may be helpful for the prognosis assessment of HSCC. Higher expression of Notch1 was associated with poor 3-year OS rates compared with low Notch1 cases. This is in concordance with previous studies in which the overexpression of Notch1 was described in HNSCC. ${ }^{20,23,24}$

Understanding the molecular mechanisms underlying HSCC initiation and tumor evolution is important to delay tumor progression and improve prognosis. To address the potential role of Notch1 signaling in the metastasis of HSCC, HSCC cell line FaDu was used and treated with the Notch1-specific siRNA to block Notch1 activity. Once the Notch1 expression was blocked by siRNA, the invasion and migration capabilities of FaDu cells were suppressed simultaneously. These results suggested that the inhibition of Notch1 may play a negative role to both the invasion and migration capabilities of FaDu cells. Our data also proved that the Notch1 signaling may be vital in the distant metastasis of HSCC.

EGFR-mediated aberrant signaling transduction is crucial in HNSCC tumorigenesis and progression. ${ }^{26}$ Previous study reported that the aberrant Notch signaling pathway interacted with other signaling pathways, such as P53, and EGFR. ${ }^{27}$ Several types of evidence indicated that Notch pathway could regulate transcription of the EGFR through TP53 $3^{20}$ and induce apoptosis through a p53-dependent pathway, which also involved in EGFR. ${ }^{28}$ As shown in our present study, we also observed a substantially downregulated EGFR mRNA and protein expression in Notch1-knockdown cells. The Notch1-related regulation of EGFR is unquestionably complex. These findings highlight the linkage between the activation of Notch signaling and EGFR, but the mechanisms of interactions between Notch and EGFR signaling in FaDu cells still require further exploration.

YBX1 is an oncogenic transcription factor and it can directly bind to the regulatory regions of EGFR. ${ }^{29}$ Previous studies have suggested that elevated expression of YBX1 consequently induced EGFR. ${ }^{30}$ In agreement with the results of EGFR expression, we demonstrated that the inhibitions of Notch1 can downregulate YBX1 and p-YBX1 expression. Given this result, these findings led us to hypothesize that YBX1 may be positively cross-linked with Notch1-regulated EGFR expression. To confirm the YBX1-dependent regulation of the EGFR, we found that ectopic expression of YBX1 resulted in substantial reversion on the suppression of EGFR expression after Notch1 knockdown. And the upregulation of YBX1 induced the invasion of FaDu. All these findings indicate that YBX1 is also a mediator of EGFR expression and cancer cell metastasis regulated by Notch1.

\section{Conclusion}

In the present study, our results demonstrated the significant overexpression of Notch1 in the HSCC cancer tissues than in the adjacent normal hypopharyngeal epithelium tissues. Further clinical feature analysis found that the high level of Notch1 expression in HSCC cancer is directly correlated with poor OS rates, distant metastasis, and tumor differentiation. The downregulation of Notch1 was able to inactivate EGFR by regulating YBX1, which resulted in the inhibition of HSCC cell invasion and migration. Together, these findings collectively demonstrate the critical role of Notch1 as a prognostic factor and a potential target to inhibit the metastasis of HSCC.

\section{Acknowledgment}

This work was supported by the Key Project of Shandong Provincial Programs for Research and Development (2018GSF118225).

\section{Disclosure}

The authors report no conflicts of interest in this work.

\section{References}

1. Yin L, Velazquez OC, Liu ZJ. Notch signaling: emerging molecular targets for cancer therapy. Biochem Pharmacol. 2010;80(5):690-701.

2. Li JL, Harris AL. Notch signaling from tumor cells: a new mechanism of angiogenesis. Cancer Cell. 2005;8(1):1-3.

3. Myong NH. Altered expressions of Notch-1 signaling proteins and betacatenin in progression of carcinoma in situ into squamous carcinoma of uterine cervix. Indian J Pathol Microbiol. 2017;60(1):21-26.

4. Li G, Zhou Z, Zhou H, et al. The expression profile and clinicopathological significance of Notch1 in patients with colorectal cancer: a meta-analysis. Future Oncol. 2017;13(23):2103-2118.

5. Hai L, Zhang C, Li T, et al. Notch1 is a prognostic factor that is distinctly activated in the classical and proneural subtype of glioblastoma and that promotes glioma cell survival via the NF- $\mathrm{KB}(\mathrm{p} 65)$ pathway. Cell Death Dis. 2018;9(2):158.

6. Hijioka H, Setoguchi T, Miyawaki A, et al. Upregulation of Notch pathway molecules in oral squamous cell carcinoma. Int J Oncol. 2010; 36(4):817-822.

7. Zhang TH, Liu HC, Zhu LJ, et al. Activation of Notch signaling in human tongue carcinoma. J Oral Pathol Med. 2011;40(1):37-45.

8. Chen C, Wang X, Huang S, Wang L, Han L, Yu S. Prognostic roles of Notch receptor mRNA expression in human ovarian cancer. Oncotarget. 2017;8(20):32731-32740.

9. Chigurupati S, Venkataraman R, Barrera D, et al. Receptor channel TRPC6 is a key mediator of Notch-driven glioblastoma growth and invasiveness. Cancer Res. 2010;70(1):418-427.

10. Sahlgren C, Gustafsson MV, Jin S, Poellinger L, Lendahl U. Notch signaling mediates hypoxia-induced tumor cell migration and invasion. Proc Natl Acad Sci U S A. 2008;105(17):6392-6397.

11. Leong KG, Niessen K, Kulic I, et al. Jagged1-mediated Notch activation induces epithelial-to-mesenchymal transition through Slug-induced repression of E-cadherin. J Exp Med. 2007;204(12):2935-2948. 
12. Hasan SS, Tsaryk R, Lange M, et al. Endothelial Notch signalling limits angiogenesis via control of artery formation. Nat Cell Biol. 2017; 19(8):928-940.

13. Zhong Y, Shen S, Zhou Y, et al. NOTCH1 is a poor prognostic factor for breast cancer and is associated with breast cancer stem cells. Onco Targets Ther. 2016;9:6865-6871.

14. Liu YF, Chiang SL, Lin CY, et al. Somatic Mutations and Genetic Variants of NOTCH1 in Head and Neck Squamous Cell Carcinoma Occurrence and Development. Sci Rep. 2016;6:24014.

15. Lee HJ, Xu X, Choe G, et al. Protein overexpression and gene amplification of epidermal growth factor receptor in nonsmall cell lung carcinomas: Comparison of four commercially available antibodies by immunohistochemistry and fluorescence in situ hybridization study. Lung Cancer. 2010;68(3):375-382.

16. Weber DC, Tille JC, Combescure C, et al. The prognostic value of expression of HIF $1 \alpha$, EGFR and VEGF-A, in localized prostate cancer for intermediate- and high-risk patients treated with radiation therapy with or without androgen deprivation therapy. Radiat Oncol. 2012;7:66

17. Zuo J, Wen M, Li S, et al. Overexpression of CXCR4 promotes invasion and migration of non-small cell lung cancer via EGFR and MMP-9. Oncol Lett. 2017;14(6):7513-7521.

18. Sheng W, Gong J, Jiang M, Zhen Y. The recombinant EGFR/CD13 bi-targeted fusion protein induces apoptosis and blocks tube formation. Oncol Rep. 2017;38(6):3507-3514.

19. Liao YH, Chiang KH, Shieh JM, et al. Epidermal growth factorinduced ANGPTL4 enhances anoikis resistance and tumour metastasis in head and neck squamous cell carcinoma. Oncogene. 2017; 36(16):2228-2242.

20. Purow BW, Sundaresan TK, Burdick MJ, et al. Notch-1 regulates transcription of the epidermal growth factor receptor through p53. Carcinogenesis. 2008;29(5):918-925.
21. Gan RH, Wei H, Xie J, et al. Notch1 regulates tongue cancer cells proliferation, apoptosis and invasion. Cell Cycle. 2018;17(2):216-224.

22. Wang Z, Banerjee S, Li Y, Rahman KM, Zhang Y, Sarkar FH. Down-regulation of notch-1 inhibits invasion by inactivation of nuclear factor-kappaB, vascular endothelial growth factor, and matrix metalloproteinase-9 in pancreatic cancer cells. Cancer Res. 2006; 66(5):2778-2784.

23. Zhao YY, Yu GT, Xiao T, Hu J. The Notch signaling pathway in head and neck squamous cell carcinoma: A meta-analysis. Adv Clin Exp Med. 2017;26(5):881-887.

24. Zhao ZL, Zhang L, Huang CF, et al. NOTCH1 inhibition enhances the efficacy of conventional chemotherapeutic agents by targeting head neck cancer stem cell. Sci Rep. 2016;6:24704.

25. Agrawal N, Frederick MJ, Pickering CR, et al. Exome sequencing of head and neck squamous cell carcinoma reveals inactivating mutations in NOTCH1. Science. 2011;333(6046):1154-1157.

26. Leeman-Neill RJ, Seethala RR, Singh SV, et al. Inhibition of EGFR-STAT3 signaling with erlotinib prevents carcinogenesis in a chemically-induced mouse model of oral squamous cell carcinoma. Cancer Prev Res (Phila). 2011;4(2):230-237.

27. Miyamoto Y, Maitra A, Ghosh B, et al. Notch mediates TGF alphainduced changes in epithelial differentiation during pancreatic tumorigenesis. Cancer Cell. 2003;3(6):565-576.

28. Yang X, Klein R, Tian X, Cheng HT, Kopan R, Shen J. Notch activation induces apoptosis in neural progenitor cells through a p53-dependent pathway. Dev Biol. 2004;269(1):81-94.

29. Sakura H, Maekawa T, Imamoto F, Yasuda K, Ishii S. Two human genes isolated by a novel method encode DNA-binding proteins containing a common region of homology. Gene. 1988;73(2):499-507.

30. Berquin IM, Pang B, Dziubinski ML, et al. Y-box-binding protein 1 confers EGF independence to human mammary epithelial cells. Oncogene. 2005;24(19):3177-3186.
OncoTargets and Therapy

\section{Publish your work in this journal}

OncoTargets and Therapy is an international, peer-reviewed, open access journal focusing on the pathological basis of all cancers, potential targets for therapy and treatment protocols employed to improve the management of cancer patients. The journal also focuses on the impact of management programs and new therapeutic agents and protocols on

\section{Dovepress}

patient perspectives such as quality of life, adherence and satisfaction. The manuscript management system is completely online and includes a very quick and fair peer-review system, which is all easy to use. Visit http://www.dovepress.com/testimonials.php to read real quotes from published authors. 